\title{
Further Result on Passivity for Discrete-Time Stochastic T-S Fuzzy Systems with Time-Varying Delays
}

\author{
Ting Lei, ${ }^{1}$ Qiankun Song, ${ }^{1}$ and Zhenjiang $\mathrm{Zhao}^{2}$ \\ ${ }^{1}$ Department of Mathematics, Chongqing Jiaotong University, Chongqing 400074, China \\ ${ }^{2}$ Department of Mathematics, Huzhou Teachers College, Huzhou 313000, China
}

Correspondence should be addressed to Qiankun Song; qiankunsong@163.com

Received 24 January 2014; Accepted 19 March 2014; Published 7 April 2014

Academic Editor: Jinde Cao

Copyright (c) 2014 Ting Lei et al. This is an open access article distributed under the Creative Commons Attribution License, which permits unrestricted use, distribution, and reproduction in any medium, provided the original work is properly cited.

\begin{abstract}
The passivity for discrete-time stochastic T-S fuzzy systems with time-varying delays is investigated. By constructing appropriate Lyapunov-Krasovskii functionals and employing stochastic analysis method and matrix inequality technique, a delay-dependent criterion to ensure the passivity for the considered T-S fuzzy systems is established in terms of linear matrix inequalities (LMIs) that can be easily checked by using the standard numerical software. An example is given to show the effectiveness of the obtained result.
\end{abstract}

\section{Introduction}

Fuzzy control offers an alternative control approach for certain nonlinear systems $[1,2]$. Among various modelbased fuzzy control approaches, the method based on TakagiSugeno (T-S) model is thought of as an effective way for the control of complex nonlinear systems, which is presented by a family of fuzzy IF-THEN rules that represent the local linear input-output relations of the system. Over the past decades, there have been significant research efforts on the stability for T-S fuzzy systems; for example, see [3-16] and references therein.

On the other hand, the passivity theory is another effective tool for the stability analysis of system. The reason is mainly twofold: (1) passivity is an expected system behavior, since the storage function induced by passivity is closely related to system energy and therefore serves as a natural candidate for Lyapunov functions and (2) stability and stabilization problems can be solved once the passivity property is assured. The passivity theory was first proposed in the circuit analysis [17] and has then been applied in many areas such as stability, signal processing, complexity, fuzzy control, chaos control, and synchronization [18-21].

Recently, some authors have studied the passivity of some systems and obtained sufficient conditions for checking the passivity of the systems that include linear systems with delays [22-24], delayed neural networks [25, 26], networked control systems [27], nonlinear discrete-time systems with direct input-output link [28], and T-S fuzzy systems [2933]. In [29], the stability of fuzzy control loops is proven with the unique condition that the controlled plant can be made passive by zero shifting. For linear time-invariant plants, this approach leads to frequency response conditions similar to the previous results in the literature but which are more general and can include robust stability considerations. In [30], the passivity and feedback passification of T-S fuzzy systems with time delays were considered. Both delayindependent and delay-dependent results were presented, and the theoretical results were given in terms of LMIs. In [31], the contiguous-time T-S fuzzy systems with timevarying delays were investigated; several criteria to ensure the passivity and feedback passification were given. In [32], the passivity and feedback passification of T-S fuzzy systems with both discrete and distributed time-varying delays were investigated without assuming the differentiability of the time-varying delays. By employing appropriate LyapunovKrasovskii functionals, several delay-dependent criteria for the passivity of the considered T-S fuzzy systems were established in terms of LMIs. In [33], the stochastic T-S fuzzy system with both discrete and distributed time-varying delays was considered; several delay-dependent criteria to ensure the passivity and passification of the considered T-S fuzzy 
systems were established. In [34], discrete-time T-S fuzzy systems with delays were considered; some sufficient conditions to verify the passivity of the uncertain discrete-time fuzzy systems were obtained. In [35], the passivity of uncertain discrete-time T-S fuzzy systems with time delays was investigated; a sufficient condition on the existence of robust passive controller was established based on the Lyapunov stability theory. In [36], stochastic discrete-time T-S fuzzy systems with delay were considered; a sufficient condition in LMIs ensuring the passivity performance of the T-S fuzzy models was presented by utilizing the Lyapunov functional method, the stochastic analysis combined with the matrix inequality techniques. In this paper, we continue to study the passivity for stochastic discrete-time T-S fuzzy systems with time-varying delay. By employing appropriate LyapunovKrasovskii functionals and stochastic analysis technique, we obtain a new delay-dependent sufficient condition for checking the passivity of the addressed T-S fuzzy systems. As pointed out in [37-39], the delay-dependent criteria have less conservatism than the delay-independent ones.

Notations. The notations are quite standard. Throughout this paper, $I$ represents the unitary matrix with appropriate dimensions; $\mathbb{N}$ stands for the set of nonnegative integers; $\mathbb{R}^{n}$ and $\mathbb{R}^{n \times m}$ denote, respectively, the $n$-dimensional Euclidean space and the set of all $n \times m$ real matrices. The superscript " $T$ " denotes matrix transposition and the asterisk “ $*$ " denotes the elements below the main diagonal of a symmetric block matrix. The notation $X \geq Y$ (resp., $X>Y$ ) means that $X$ and $Y$ are symmetric matrices and that $X-Y$ is positive semidefinite (resp., positive definite). Also $\|\cdot\|$ is the Euclidean norm in $\mathbb{R}^{n}$. And $\lambda_{\text {min }}(A)$ (resp., $\lambda_{\max }(A)$ ) denotes the least (resp., largest) eigenvalue of symmetric matrix $A$. For a positive constant $a,[a]$ denotes the integer part of $a$. For integers $a, b$ with $a<b, \mathbb{N}[a, b]$ denotes the discrete interval given by $\mathbb{N}[a, b]=\{a, a+1, \ldots, b-$ $1, b\}$. Also $C\left(\mathbb{N}[-\tau, 0], \mathbb{R}^{n}\right)$ denotes the set of all functions $\phi: \mathbb{N}[-\tau, 0] \rightarrow \mathbb{R}^{n}$. Let $\left(\Omega, \mathscr{F},\{\mathscr{F}\}_{t \geq 0}, \mathscr{P}\right)$ be a complete probability space with filtration $\{\mathscr{F}\}_{t \geq 0}$ satisfying the usual conditions (i.e., it is right continuous and $\mathscr{F}_{0}$ contains all $\mathscr{P}$-null sets). $\mathbb{E}\{\cdot\}$ stands for the mathematical expectation operator with respect to the given probability measure $\mathscr{P}$. Denote by $L_{\mathscr{F}_{0}}^{2}\left(\mathbb{N}[-\tau, 0], \mathbb{R}^{n}\right)$ the family of all $\mathscr{F}_{0}$-measurable $C\left(\mathbb{N}[-\tau, 0], \mathbb{R}^{n}\right)$ valued random variables $\psi=\{\psi(s): s \in$ $\mathbb{N}[-\tau, 0]\}$ such that $\sup _{s \in \mathbb{N}[-\tau, 0]} \mathbb{E}\{|\psi(s)|\}<\infty . \Delta V(k)$ denotes the difference of function $V(k)$ given by $\Delta V(k)=V(k+1)-$ $V(k)$. Matrices, if not explicitly specified, are assumed to have compatible dimensions.

\section{Model Description and Preliminaries}

In this section, we consider a discrete-time T-S fuzzy system with stochastic disturbances and time-varying delay with the $i$ th rule formulated in the following form.

Plant Rule $i$. If $z_{1}(t)$ is $M_{i 1}$ and ... and $z_{p}(t)$ is $M_{i p}$, then

$$
\begin{aligned}
x(k+1)= & A_{i} x(k)+B_{i} x(k-\tau(k)) \\
& +U_{i} J(k)+\sigma_{i}(x(k), x(k-\tau(k))) \omega_{i}(k),
\end{aligned}
$$

$$
\begin{aligned}
& y(k)=C_{i} x(k)+D_{i} x(k-\tau(k))+V_{i} J(k), \\
& x(k)=\varphi(k), \quad k \in \mathbb{N}[-\bar{\tau}, 0]
\end{aligned}
$$

for $k \in \mathbb{N}, i=1,2, \ldots, r$, and $r$ is the number of IFTHEN rules, where $x(k) \in \mathbb{R}^{n}$ is the state vector, $J(k) \in$ $\mathbb{R}^{m}$ is a deterministic exogenous input, $y(k) \in \mathbb{R}^{m}$ is the measurement output vector, $A_{i}, B_{i}, U_{i}, C_{i}, D_{i}$, and $V_{i}$ are system matrices with compatible dimensions, the positive integer $\tau(k)$ corresponds to the transmission delay and satisfies $\tau \leq \tau(k) \leq \bar{\tau}$ ( $\tau \geq 0$ and $\bar{\tau} \geq 0$ are known integers), $\sigma \in \mathbb{R}^{n}$ is the diffusion coefficient vector, and $\omega_{i}(k)$ is a scalar Brownian motion defined on $(\Omega, \mathscr{F}, \mathscr{P})$ with $\mathbb{E}\left\{\omega_{i}(k)\right\}=0$, $\mathbb{E}\left\{\omega_{i}^{2}(k)\right\}=1$ and $\mathbb{E}\left\{\omega_{i}(l) \omega_{i}(k)\right\}=0(l \neq k)$.

Let $\mu_{i}(k)$ be the normalized membership function of the inferred fuzzy set $\gamma_{i}(k)$; that is,

$$
\mu_{i}(k)=\frac{\gamma_{i}(k)}{\sum_{i=1}^{r} \gamma_{i}(k)},
$$

where $\gamma_{i}(k)=\prod_{j=1}^{p} M_{i j}\left(z_{j}(k)\right)$ with $M_{i j}\left(z_{j}(k)\right)$ being the grade of membership function of $z_{j}(k)$ in $M_{i j}(k)$. It is assumed that $\gamma_{i}(k) \geq 0(i=1,2, \ldots, r)$ and $\sum_{i=1}^{r} \gamma_{i}(k)>0$ for all $k$. Thus $\mu_{i}(k) \geq 0$ and $\sum_{i=1}^{r} \mu_{i}(k)=1$ for all $k$. And the T-S fuzzy model (1) can be represented as

$$
\begin{aligned}
x(k+1) & =\sum_{i=1}^{r} \mu_{i}(t)\left[A_{i} x(k)+B_{i} x(k-\tau(k))+U_{i} J(k)\right. \\
& \left.+\sigma_{i}(x(k), x(k-\tau(k))) \omega_{i}(k)\right], \\
y(k)= & \sum_{i=1}^{r} \mu_{i}(t)\left[C_{i} x(k)+D_{i} x(k-\tau(k))+V_{i} J(k)\right], \\
x(k) & =\varphi(k), \quad k \in \mathbb{N}[-\bar{\tau}, 0] .
\end{aligned}
$$

In the literature, different definitions of passivity have been used. Taking into account the stochastic nature of the T-S fuzzy systems considered in this paper, we adopt the definition in [36].

Definition 1. System (1) is called passive in the sense of expectation if there exists a scalar $\gamma>0$ such that

$$
2 \sum_{k=0}^{T} \mathbb{E}\left\{y^{T}(k) J(k)\right\} \geq-\gamma \sum_{k=0}^{T} \mathbb{E}\left\{J^{T}(k) J(k)\right\}
$$

for all integers $T$ and the solution of (1) with $\phi(\cdot) \equiv 0$.

Throughout this paper, we make the following assumption.

Assumption 2 (see [36]). There exist matrices $W_{i}$ and $S_{i}$ such that

$$
\begin{array}{r}
\sigma_{i}^{T}(x, y) \sigma_{i}(x, y) \leq\left\|W_{i} x\right\|^{2}+\left\|S_{i} y\right\|^{2}, \\
\forall x, y \in \mathbb{R}^{n}, \quad i=1,2, \ldots, r .
\end{array}
$$

In obtaining the main result of this paper, the following lemma will be useful for the proof. 
Lemma 3 (see [40]). Suppose that matrices $M_{i} \in \mathbb{R}^{n \times m}$ ( $i=$ $1,2, \ldots, s)$ and a positive-semidefinite matrix $P \in \mathbb{R}^{n \times n}$ are given. If $\sum_{i=1}^{s} h_{i}=1$ and $0 \leq h \leq 1$, then

$$
\left(\sum_{i=1}^{s} h_{i} M_{i}\right)^{T} P\left(\sum_{i=1}^{s} h_{i} M_{i}\right) \leq \sum_{i=1}^{s} h_{i} M_{i}^{T} P M_{i} .
$$

\section{Main Results}

In this section, we will establish our main criterion based on the LMI approach. For presentation convenience, in the following, we denote that $\delta=[(\tau+\bar{\tau}) / 2]$.

Theorem 4. Under Assumption 2, model (1) is passive in the sense of Definition 1 if there exist two scalars $\gamma>0$ and $\lambda>0$ and eight symmetric positive definite matrices $P, Q_{1}, Q_{2}, Q_{3}$, $Q_{4}, R_{1}, R_{2}$, and $R_{3}$ such that the following LMIs hold for $i=$ $1,2, \ldots, r$ :

$$
\begin{gathered}
P+R<\lambda I, \\
\Pi^{(i)}+\Omega_{1}<0, \\
\Pi^{(i)}+\Omega_{2}<0
\end{gathered}
$$

hold or

$$
\begin{gathered}
P+R<\lambda I, \\
\Pi^{(i)}+\Omega_{3}<0, \\
\Pi^{(i)}+\Omega_{4}<0
\end{gathered}
$$

hold, where

$$
\begin{aligned}
\Pi^{(i)} & =\left[\begin{array}{cccccc}
\Pi_{11}^{(i)} & \Pi_{12}^{(i)} & 0 & 0 & 0 & \Pi_{16}^{(i)} \\
* & \Pi_{22}^{(i)} & 0 & 0 & 0 & \Pi_{26}^{(i)} \\
* & * & -Q_{1} & 0 & 0 & 0 \\
* & * & * & -Q_{2} & 0 & 0 \\
* & * & * & * & -Q_{3} & 0 \\
* & * & * & * & * & \Pi_{66}^{(i)}
\end{array}\right], \\
\Omega_{1} & =\left[\begin{array}{ccccccc}
-R_{1} & 0 & 0 & R_{1} & 0 & 0 \\
* & -3 R_{2} & 2 R_{2} & R_{2} & 0 & 0 \\
* & * & -2 R_{2}-R_{3} & 0 & R_{3} & 0 \\
* & * & * & -R_{1}-R_{2} & 0 & 0 \\
* & * & * & * & -R_{3} & 0 \\
* & * & * & * & * & 0
\end{array}\right], \\
\Omega_{2} & =\left[\begin{array}{cccccc}
-R_{1} & 0 & 0 & R_{1} & 0 & 0 \\
* & -3 R_{2} & R_{2} & 2 R_{2} & 0 & 0 \\
* & * & -R_{2}-R_{3} & 0 & R_{3} & 0 \\
* & * & * & -R_{1}-2 R_{2} & 0 & 0 \\
* & * & * & * & -R_{3} & 0 \\
* & * & * & * & * & 0
\end{array}\right],
\end{aligned}
$$

$$
\begin{array}{r}
\Omega_{3}=\left[\begin{array}{cccccc}
-R_{1} & 0 & 0 & R_{1} & 0 & 0 \\
* & -3 R_{3} & R_{3} & 0 & 2 R_{3} & 0 \\
* & * & -R_{2}-R_{3} & R_{2} & 0 & 0 \\
* & * & * & -R_{1}-R_{2} & 0 & 0 \\
* & * & * & * & -2 R_{3} & 0 \\
* & * & * & * & * & 0
\end{array}\right], \\
\Omega_{4}=\left[\begin{array}{cccccc}
-R_{1} & 0 & 0 & R_{1} & 0 & 0 \\
* & -3 R_{3} & 2 R_{3} & 0 & R_{3} & 0 \\
* & * & -R_{2}-2 R_{3} & R_{2} & 0 & 0 \\
* & * & * & -R_{1}-R_{2} & 0 & 0 \\
* & * & * & * & -R_{3} & 0 \\
* & * & * & * & * & 0
\end{array}\right],
\end{array}
$$

in which $R=\tau^{2} R_{1}+(\delta-\tau)^{2} R_{2}+(\bar{\tau}-\delta)^{2} R_{3}, \Pi_{11}^{(i)}=A_{i}^{T}(P+$ R) $A_{i}-P+Q_{1}+Q_{2}+Q_{3}+(1+\bar{\tau}-\tau) Q_{4}-R A_{i}-A_{i}^{T} R+R+\lambda W_{i}^{T} W_{i}$, $\Pi_{12}^{(i)}=A_{i}^{T}(P+R) B_{i}-R B_{i}, \Pi_{16}^{(i)}=A_{i}^{T}(P+R) U_{i}-R U_{i}-C_{i}^{T}$, $\Pi_{22}^{(i)}=B_{i}^{T}(P+R) B_{i}-Q_{4}+\lambda S_{i}^{T} S_{i}, \Pi_{26}^{(i)}=B_{i}^{T}(P+R) U_{i}-D_{i}^{T}$, and $\Pi_{66}^{(i)}=U_{i}^{T}(P+R) U_{i}-V_{i}-V_{i}^{T}-\gamma I$.

Proof. Defining $\eta(k)=x(k+1)-x(k)$, we consider the following Lyapunov-Krasovskii functional candidate for model (1) as

$$
V(k)=\sum_{i=1}^{4} V_{i}(k),
$$

where

$$
\begin{aligned}
V_{1}(k)= & x^{T}(k) P x(k), \\
V_{2}(k)= & \sum_{i=k-\delta}^{k-1} x^{T}(i) Q_{1} x(i)+\sum_{i=k-\tau}^{k-1} x^{T}(i) Q_{2} x(i) \\
& +\sum_{i=k-\bar{\tau}}^{k-1} x^{T}(i) Q_{3} x(i), \\
V_{3}(k)= & \sum_{i=k-\tau(k)}^{k-1} x^{T}(i) Q_{4} x(i)+\sum_{l=k-\bar{\tau}+1}^{k-\tau} \sum_{i=l}^{k-1} x^{T}(i) Q_{4} x(i), \\
V_{4}(k)= & \tau \sum_{l=k-\tau}^{k-1} \sum_{i=l}^{k-1} \eta^{T}(i) R_{1} \eta(i) \\
& +(\delta-\tau) \sum_{l=k-\delta}^{k-\tau-1} \sum_{i=l}^{k-1} \eta^{T}(i) R_{2} \eta(i) \\
& +(\bar{\tau}-\delta) \sum_{l=k-\bar{\tau}}^{k-\delta-1} \sum_{i=l}^{k-1} \eta^{T}(i) R_{3} \eta(i) .
\end{aligned}
$$

Calculating the difference of $V_{i}(k)(i=1,2,3,4)$ along the trajectories of model (1) and taking the mathematical expectation, we obtain that

$$
\begin{aligned}
\mathbb{E} & \left\{\Delta V_{1}(k)\right\} \\
& =\mathbb{E}\left\{\left(\sum _ { i = 1 } ^ { r } \mu _ { i } ( t ) \left[A_{i} x(k)+B_{i} x(k-\tau(k))+U_{i} J(k)\right.\right.\right.
\end{aligned}
$$




$$
\begin{gathered}
\left.\left.+\sigma_{i}(x(k), x(k-\tau(k))) \omega_{i}(k)\right]\right)^{T} P \\
\times\left(\sum _ { i = 1 } ^ { r } \mu _ { i } ( t ) \left[A_{i} x(k)+B_{i} x(k-\tau(k))+U_{i} J(k)\right.\right. \\
\left.\left.+\sigma_{i}(x(k), x(k-\tau(k))) \omega_{i}(k)\right]\right) \\
\left.-x^{T}(k) P x(k)\right\} \\
\leq \mathbb{E}\left\{\sum _ { i = 1 } ^ { r } \mu _ { i } ( t ) \left[A_{i} x(k)+B_{i} x(k-\tau(k))+U_{i} J(k)\right.\right. \\
\left.+\sigma_{i}(x(k), x(k-\tau(k))) \omega_{i}(k)\right]^{T} P \\
\times\left[A_{i} x(k)+B_{i} x(k-\tau(k))+U_{i} J(k)\right. \\
\left.\left.\left.+\sigma_{i}(x), x(k-\tau(k))\right) \omega_{i}(k)\right]-x^{T}(k) P x(k)\right\} \\
=\mathbb{E}\left\{\sum _ { i = 1 } ^ { r } \mu _ { i } ( t ) \left[x^{T}(k)\left(A_{i}^{T} P A_{i}-P\right) x(k)\right.\right. \\
+2 x^{T}(k) A_{i}^{T} P B_{i} x(k-\tau(k)) \\
+2 x^{T}(k) A_{i}^{T} P U_{i} J(k) \\
+x^{T}(k-\tau(k)) B_{i}^{T} P B_{i} x(k-\tau(k)) \\
+2 x^{T}(k-\tau(k)) B_{i}^{T} P U_{i} J(k) \\
+J^{T}(k) U_{i}^{T} P U_{i} J(k) \\
+\sigma_{i}^{T}(x(k), x(k-\tau(k))) \\
\times
\end{gathered}
$$

$$
\begin{aligned}
\mathbb{E}\{\Delta & \left.V_{2}(k)\right\} \\
=\mathbb{E} & \left\{x^{T}(k)\left(Q_{1}+Q_{2}+Q_{3}\right) x(k)\right. \\
& -x^{T}(k-\delta) Q_{1} x(k-\delta)-x^{T}(k-\tau) Q_{2} x(k-\tau) \\
& \left.-x^{T}(k-\bar{\tau}) Q_{3} x(k-\bar{\tau})\right\},
\end{aligned}
$$

$$
\begin{aligned}
\mathbb{E}\{\Delta & \left.V_{3}(k)\right\} \\
=\mathbb{E} & \left\{\sum_{i=k+1-\tau(k+1)}^{k-\tau} x^{T}(i) Q_{4} x(i)+\sum_{i=k-\tau+1}^{k-1} x^{T}(i) Q_{4} x(i)\right. \\
& +x^{T}(k) Q_{4} x(k)-\sum_{i=k-\tau(k)+1}^{k-1} x^{T}(i) Q_{4} x(i) \\
& -x^{T}(k-\tau(k)) Q_{4} x(k-\tau(k)) \\
& \left.+(\bar{\tau}-\tau) x^{T}(k) Q_{4} x(k)-\sum_{l=k-\bar{\tau}+1}^{k-\tau} x^{T}(l) Q_{4} x(l)\right\} \\
\leq \mathbb{E} & \left\{(1+\bar{\tau}-\tau) x^{T}(k) Q_{4} x(k)\right. \\
& \left.-x^{T}(k-\tau(k)) Q_{4} x(k-\tau(k))\right\}
\end{aligned}
$$

$$
\begin{aligned}
\mathbb{E}\{ & \left.\Delta V_{4}(k)\right\} \\
=\mathbb{E} & \left\{\eta^{T}(k) R \eta(k)-\tau \sum_{i=k-\tau}^{k-1} \eta^{T}(i) R_{1} \eta(i)\right. \\
& -(\delta-\tau) \sum_{i=k-\delta}^{k-\tau-1} \eta^{T}(i) R_{2} \eta(i) \\
& \left.-(\bar{\tau}-\delta) \sum_{i=k-\bar{\tau}}^{k-\delta-1} \eta^{T}(i) R_{3} \eta(i)\right\}
\end{aligned}
$$

In deriving inequalities (15) and (17), Lemma 3 and the condition $\tau \leq \tau(k) \leq \bar{\tau}$ have been used, respectively.

From the definition of $\eta(k)$ and (3) and applying Lemma 3, we have

$$
\begin{aligned}
& \mathbb{E}\left\{\eta^{T}(k) R \eta(k)\right\} \\
& \leq \mathbb{E}\left\{\sum _ { i = 1 } ^ { r } \mu _ { i } ( t ) \left[x^{T}(k)\left(A_{i}^{T} R A_{i}-R A_{i}-A_{i}^{T} R+R\right) x(k)\right.\right. \\
&+2 x^{T}(k)\left(A_{i}^{T} R B_{i}-R B_{i}\right) x(k-\tau(k)) \\
&+2 x^{T}(k)\left(A_{i}^{T} R U_{i}-R U_{i}\right) J(k) \\
&+x^{T}(k-\tau(k)) B_{i}^{T} R B_{i} x(k-\tau(k)) \\
&+2 x^{T}(k-\tau(k)) B_{i}^{T} R U_{i} J(k) \\
&+J^{T}(k) U_{i}^{T} R U_{i} J(k) \\
&+\sigma_{i}^{T}(x(k), x(k-\tau(k))) \\
&\left.\left.\times R \sigma_{i}(x(k), x(k-\tau(k)))\right]\right\} .
\end{aligned}
$$

It is easy to get

$$
\begin{aligned}
& -\tau \sum_{i=k-\tau}^{k-1} \eta^{T}(i) R_{1} \eta(i) \\
& \leq-\sum_{i=k-\tau}^{k-1} \eta^{T}(i) R_{1} \sum_{i=k-\tau}^{k-1} \eta(i) \\
& =\left[\begin{array}{c}
x(k) \\
x(k-\tau)
\end{array}\right]^{T}\left[\begin{array}{cc}
-R_{1} & R_{1} \\
* & -R_{1}
\end{array}\right]\left[\begin{array}{c}
x(k) \\
x(k-\tau)
\end{array}\right] .
\end{aligned}
$$

When $\tau \leq \tau(k) \leq \delta$, let $a(k)=(\tau(k)-\tau) /(\delta-\tau)$; then $0 \leq a(k) \leq 1$. It is easy to get that

$$
\begin{aligned}
& -(\delta-\tau) \sum_{i=k-\delta}^{k-\tau-1} \eta^{T}(i) R_{2} \eta(i) \\
& =-(\delta-\tau) \sum_{i=k-\delta}^{k-\tau(k)-1} \eta^{T}(i) R_{2} \eta(i)
\end{aligned}
$$




$$
\begin{aligned}
& -(\delta-\tau) \sum_{i=k-\tau(k)}^{k-\tau-1} \eta^{T}(i) R_{2} \eta(i) \\
& =-(\delta-\tau(k)) \sum_{i=k-\delta}^{k-\tau(k)-1} \eta^{T}(i) R_{2} \eta(i) \\
& -(\tau(k)-\tau) \sum_{i=k-\delta}^{k-\tau(k)-1} \eta^{T}(i) R_{2} \eta(i) \\
& -(\delta-\tau(k)) \sum_{i=k-\tau(k)}^{k-\tau-1} \eta^{T}(i) R_{2} \eta(i) \\
& -(\tau(k)-\tau) \sum_{i=k-\tau(k)}^{k-\tau-1} \eta^{T}(i) R_{2} \eta(i) \\
& \leq-(\delta-\tau(k)) \sum_{i=k-\delta}^{k-\tau(k)-1} \eta^{T}(i) R_{2} \eta(i) \\
& -a(k)(\delta-\tau(k)) \sum_{i=k-\delta}^{k-\tau(k)-1} \eta^{T}(i) R_{2} \eta(i) \\
& -(1-a(k))(\tau(k)-\tau) \sum_{i=k-\tau(k)}^{k-\tau-1} \eta^{T}(i) R_{2} \eta(i) \\
& -(\tau(k)-\tau) \sum_{i=k-\tau(k)}^{k-\tau-1} \eta^{T}(i) R_{2} \eta(i) \\
& \leq-\sum_{i=k-\delta}^{k-\tau(k)-1} \eta^{T}(i) R_{2} \sum_{i=k-\delta}^{k-\tau(k)-1} \eta(i) \\
& -a(k) \sum_{i=k-\delta}^{k-\tau(k)-1} \eta^{T}(i) R_{2} \sum_{i=k-\delta}^{k-\tau(k)-1} \eta(i) \\
& -(1-a(k)) \sum_{i=k-\tau(k)}^{k-\tau-1} \eta^{T}(i) R_{2} \sum_{i=k-\tau(k)}^{k-\tau-1} \eta(i) \\
& -\sum_{i=k-\tau(k)}^{k-\tau-1} \eta^{T}(i) R_{2} \sum_{i=k-\tau(k)}^{k-\tau-1} \eta(i) \\
& =\left[\begin{array}{c}
x(k-\tau(k)) \\
x(k-\delta) \\
x(k-\tau)
\end{array}\right]^{T}\left[\begin{array}{ccc}
-2 R_{2} & R_{2} & R_{2} \\
* & -R_{2} & 0 \\
* & * & -R_{2}
\end{array}\right]\left[\begin{array}{c}
x(k-\tau(k)) \\
x(k-\delta) \\
x(k-\tau)
\end{array}\right] \\
& +a(k)\left[\begin{array}{c}
x(k-\tau(k)) \\
x(k-\delta)
\end{array}\right]^{T}\left[\begin{array}{cc}
-R_{2} & R_{2} \\
* & -R_{2}
\end{array}\right]\left[\begin{array}{c}
x(k-\tau(k)) \\
x(k-\delta)
\end{array}\right] \\
& +(1-a(k))\left[\begin{array}{c}
x(k-\tau(k)) \\
x(k-\tau)
\end{array}\right]^{T} \\
& \times\left[\begin{array}{cc}
-R_{2} & R_{2} \\
* & -R_{2}
\end{array}\right]\left[\begin{array}{c}
x(k-\tau(k)) \\
x(k-\tau)
\end{array}\right],
\end{aligned}
$$

$$
\begin{aligned}
& -(\bar{\tau}-\delta) \sum_{i=k-\bar{\tau}}^{k-\delta-1} \eta^{T}(i) R_{3} \eta(i) \\
& \leq\left[\begin{array}{c}
x(k-\delta) \\
x(k-\bar{\tau})
\end{array}\right]^{T}\left[\begin{array}{cc}
-R_{3} & R_{3} \\
* & -R_{3}
\end{array}\right]\left[\begin{array}{c}
x(k-\delta) \\
x(k-\bar{\tau})
\end{array}\right] .
\end{aligned}
$$

From the first inequality of condition (7) and Assumption 2, we get

$$
\begin{aligned}
& \sigma_{i}^{T}(x(k), x(k-\tau(k)))(P+R) \sigma_{i}(x(k), x(k-\tau(k))) \\
& \quad \leq \lambda\left[\left\|W_{i} x(k)\right\|^{2}+\left\|S_{i} x(k-\tau(k))\right\|^{2}\right] .
\end{aligned}
$$

Denote that $\alpha(k)=\left(x^{T}(k), x^{T}(k-\tau(k)), x^{T}(k-\delta), x^{T}(k-\right.$ $\left.\tau), x^{T}(k-\bar{\tau}), J^{T}(k)\right)^{T}$. It follows from (15) to (23) that

$$
\begin{aligned}
& \mathbb{E}\left\{\Delta V(k)-2 y^{T}(k) J(k)-\gamma J^{T}(k) J(k)\right\} \\
& \leq \mathbb{E}\left\{\sum _ { i = 1 } ^ { r } \mu _ { i } ( t ) \alpha ^ { T } ( k ) \left[a(k)\left(\Pi^{(i)}+\Omega_{1}\right)+(1-a(k))\right.\right. \\
&\left.\left.\times\left(\Pi^{(i)}+\Omega_{2}\right)\right] \alpha(k)\right\} .
\end{aligned}
$$

From the second inequality and the third inequality of condition (7), we get

$$
\mathbb{E}\left\{\Delta V(k)-2 y^{T}(k) J(k)-\gamma J^{T}(k) J(k)\right\} \leq 0
$$

and therefore we have

$$
\begin{aligned}
& 2 \sum_{k=0}^{T} \mathbb{E}\left\{y^{T}(k) J(k)\right\} \\
& \quad \geq \sum_{k=0}^{T} \mathbb{E}\{\Delta V(k)\}-\gamma \sum_{k=0}^{T} \mathbb{E}\left\{J^{T}(k) J(k)\right\} \\
& \quad=\sum_{k=0}^{T} \mathbb{E}\{V(T)-V(0)\}-\gamma \sum_{k=0}^{T} \mathbb{E}\left\{J^{T}(k) J(k)\right\} \\
& \quad \geq-\sum_{k=0}^{T} \mathbb{E}\{V(0)\}-\gamma \sum_{k=0}^{T} \mathbb{E}\left\{J^{T}(k) J(k)\right\} \\
& \quad=-\gamma \sum_{k=0}^{T} \mathbb{E}\left\{J^{T}(k) J(k)\right\}
\end{aligned}
$$

for all integers $T \geq 0$. From Definition 1, we know that (26) implies that the stochastic T-S fuzzy system (1) is globally passive in the sense of expectation. 
When $\delta \leq \tau(t) \leq \bar{\tau}$, let $b(k)=(\bar{\tau}-\tau(k)) /(\bar{\tau}-\delta)$; then $0 \leq b(k) \leq 1$. In the similitude of the proof of inequality (21), we have

$$
\begin{aligned}
& -(\bar{\tau}-\delta) \sum_{i=k-\bar{\tau}}^{k-\delta-1} \eta^{T}(i) R_{3} \eta(i) \\
& \leq\left[\begin{array}{c}
x(k-\tau(k)) \\
x(k-\bar{\tau}) \\
x(k-\delta)
\end{array}\right]^{T}\left[\begin{array}{ccc}
-2 R_{3} & R_{3} & R_{3} \\
* & -R_{3} & 0 \\
* & * & -R_{3}
\end{array}\right]\left[\begin{array}{c}
x(k-\tau(k)) \\
x(k-\bar{\tau}) \\
x(k-\delta)
\end{array}\right] \\
& +b(k)\left[\begin{array}{c}
x(k-\tau(k)) \\
x(k-\bar{\tau})
\end{array}\right]^{T}\left[\begin{array}{cc}
-R_{3} & R_{3} \\
* & -R_{3}
\end{array}\right]\left[\begin{array}{c}
x(k-\tau(k)) \\
x(k-\bar{\tau})
\end{array}\right] \\
& +(1-b(k))\left[\begin{array}{c}
x(k-\tau(k)) \\
x(k-\delta)
\end{array}\right]^{T} \\
& \times\left[\begin{array}{cc}
-R_{3} & R_{3} \\
* & -R_{3}
\end{array}\right]\left[\begin{array}{c}
x(k-\tau(k)) \\
x(k-\delta)
\end{array}\right], \\
& -(\delta-\tau) \sum_{i=k-\delta}^{k-\tau-1} \eta^{T}(i) R_{2} \eta(i) \\
& \leq\left[\begin{array}{c}
x(k-\tau) \\
x(k-\delta)
\end{array}\right]^{T}\left[\begin{array}{cc}
-R_{2} & R_{2} \\
* & -R_{2}
\end{array}\right]\left[\begin{array}{c}
x(k-\tau) \\
x(k-\delta)
\end{array}\right]
\end{aligned}
$$

It follows from (15)-(20), (23), (27), and (28) that

$$
\begin{gathered}
\mathbb{E}\left\{\Delta V(k)-2 y^{T}(k) J(k)-\gamma J^{T}(k) J(k)\right\} \\
\leq \mathbb{E}\left\{\sum _ { i = 1 } ^ { r } \mu _ { i } ( t ) \alpha ^ { T } ( k ) \left[b(k)\left(\Pi^{(i)}+\Omega_{3}\right)+(1-b(k))\right.\right. \\
\left.\left.\times\left(\Pi^{(i)}+\Omega_{4}\right)\right] \alpha(k)\right\} .
\end{gathered}
$$

From the second inequality and the third inequality of condition (8), we get

$$
\mathbb{E}\left\{\Delta V(k)-2 y^{T}(k) J(k)-\gamma J^{T}(k) J(k)\right\} \leq 0 .
$$

By using same method in (25) and (26), we know that the stochastic T-S fuzzy system (1) is globally passive in the sense of expectation. The proof is completed.

Remark 5. In [36], the passivity of discrete-time stochastic T-S fuzzy systems was investigated. Compared with the approach of [36], the method in this paper has two advantages: (i) different from the method of [36], the variation interval of the time-varying delays $\tau(k)$ is divided into two subintervals, and the variation of the LyapunovKrasovskii functional is checked for the cases when the timevarying delay varies in the subintervals; (ii) the terms $-(\delta-$ $\tau) \sum_{i=k-\delta}^{k-\tau-1} \eta^{T}(i) R_{2} \eta(i)$ and $-(\bar{\tau}-\delta) \sum_{i=k-\bar{\tau}}^{k-\delta-1} \eta^{T}(i) R_{3} \eta(i)$ are both divided into four parts and two scalars $a(k)$ and $b(k)$ are introduced, respectively. From above-mentioned techniques, we know that more information of the time-varying delays $\tau(k)$ was employed in deriving the result of this paper. Thus, the obtained result in this paper is expected to have less conservatism than the result in [36].

\section{Example}

Example 1. Consider a T-S fuzzy system (1) with $r=2$, where $r$ is the number of IF-THEN rules. The time-varying delay $\tau(k)$ is assumed to have $\tau=2$ and $\bar{\tau}=5$. Other parameters are given as follows:

$$
\begin{array}{rlrl}
A_{1} & =\left[\begin{array}{cc}
0.04 & 0.01 \\
0.01 & -0.02
\end{array}\right], & B_{1}=\left[\begin{array}{cc}
-0.02 & 0.01 \\
0.01 & 0.03
\end{array}\right], \\
U_{1}=\left[\begin{array}{cc}
-0.01 & 0.02 \\
0.01 & 0.01
\end{array}\right], & C_{1}=\left[\begin{array}{cc}
0 & 0.02 \\
-0.01 & 0.01
\end{array}\right], \\
D_{1}=\left[\begin{array}{cc}
0.04 & 0.01 \\
-0.02 & -0.01
\end{array}\right], & V_{1}=\left[\begin{array}{cc}
-0.01 & -0.02 \\
0 & 0.01
\end{array}\right], \\
A_{2}=\left[\begin{array}{cc}
0.01 & 0.03 \\
0 & 0.01
\end{array}\right], & B_{2}=\left[\begin{array}{cc}
-0.01 & 0.02 \\
-0.02 & 0.03
\end{array}\right], \\
U_{2}=\left[\begin{array}{cc}
-0.01 & 0.02 \\
0.01 & 0.01
\end{array}\right], & C_{2}=\left[\begin{array}{cc}
0.01 & 0 \\
0.02 & 0.01
\end{array}\right], \\
D_{2}=\left[\begin{array}{cc}
-0.01 & -0.01 \\
0.01 & 0.02
\end{array}\right], & V_{2}=\left[\begin{array}{cc}
0.01 & -0.01 \\
0.01 & 0.02
\end{array}\right], \\
W_{1}=\left[\begin{array}{cc}
-0.01 & 0.01 \\
0.02 & 0
\end{array}\right], & S_{1}=\left[\begin{array}{cc}
0.02 & 0 \\
0.01 & -0.01
\end{array}\right], \\
W_{2}=\left[\begin{array}{cc}
0.01 & 0 \\
0.02 & 0.05
\end{array}\right], & S_{2}=\left[\begin{array}{cc}
0.01 & -0.01 \\
0.01 & 0.01
\end{array}\right] .
\end{array}
$$

By using the Matlab LMI Control Toolbox, we can find a solution to the LMIs in (7) as follows:

$$
\begin{gathered}
P=10^{4}\left[\begin{array}{cc}
1.0868 & -0.0007 \\
-0.0007 & 1.0878
\end{array}\right] \\
Q_{1}=10^{3}\left[\begin{array}{cc}
1.3480 & -0.0014 \\
-0.0014 & 1.3499
\end{array}\right] \\
Q_{2}=10^{3}\left[\begin{array}{cc}
1.3488 & -0.0011 \\
-0.0011 & 1.3501
\end{array}\right] \\
Q_{3}=10^{3}\left[\begin{array}{cc}
1.3489 & -0.0009 \\
-0.0009 & 1.3502
\end{array}\right] \\
Q_{4}=10^{3}\left[\begin{array}{cc}
1.3603 & -0.0037 \\
-0.0037 & 1.3639
\end{array}\right] \\
R_{1}=\left[\begin{array}{cc}
1.7200 & 0.8232 \\
0.8232 & 0.5407
\end{array}\right] \\
R_{2}=\left[\begin{array}{ll}
0.2118 & 0.1525 \\
0.1525 & 0.1121
\end{array}\right] \\
R_{3}=\left[\begin{array}{ll}
1.8563 & 0.8703 \\
0.8703 & 0.5674
\end{array}\right] \\
\gamma=1.3546 \times 10^{3}, \quad \lambda=1.2234 \times 10^{4}
\end{gathered}
$$

According to Theorem 4, the considered model (1) is passive in the sense of Definition 1. 


\section{Conclusions}

In this paper, the passivity for discrete-time stochastic T-S fuzzy systems with time-varying delays has been investigated. By constructing appropriate Lyapunov-Krasovskii functionals and employing stochastic analysis method and matrix inequality technique, a delay-dependent criterion to ensure the passivity for the considered T-S fuzzy systems has been established in terms of linear matrix inequalities (LMIs) that can be easily checked by using the standard numerical software. An example is also given to show the effectiveness of the obtained result.

\section{Conflict of Interests}

The authors declare that there is no conflict of interests regarding the publication of this paper.

\section{Acknowledgments}

The authors would like to thank the editor and the reviewers for their valuable suggestions and comments which have led to a much improved paper. This work was supported by the National Natural Science Foundation of China under Grants 61273021 and 11172247 and in part by the Natural Science Foundation Project of CQ cstc2013jjB40008.

\section{References}

[1] T. Takagi and M. Sugeno, "Fuzzy identification of systems and its applications to modeling and control," IEEE Transactions on Systems, Man and Cybernetics, vol. 15, no. 1, pp. 116-132, 1985.

[2] G. Feng, "A survey on analysis and design of model-based fuzzy control systems," IEEE Transaction on Fuzzy Systems, vol. 14, no. 5, pp. 676-697, 2006.

[3] K. Kiriakidis, A. Grivas, and A. Tzes, "Quadratic stability analysis of the Takagi-Sugeno fuzzy model," Fuzzy Sets and Systems, vol. 98, no. 1, pp. 1-14, 1998.

[4] F. Cuesta, F. Gordillo, J. Aracil, and A. Ollero, "Stability analysis of nonlinear multivariable Takagi-Sugeno fuzzy control systems," IEEE Transaction on Fuzzy Systems, vol. 7, no. 5, pp. 508$520,1999$.

[5] J.-H. Chou and S.-H. Chen, "Stability analysis of the discrete Takagi-Sugeno fuzzy model with time-varying consequent uncertainties," Fuzzy Sets and Systems, vol. 118, no. 2, pp. 271279, 2001.

[6] C. Pang and S. Guu, "Sufficient conditions for the stability of linear Takagi-Sugeno free fuzzy systems," IEEE Transaction on Fuzzy Systems, vol. 11, no. 5, pp. 695-700, 2003.

[7] Z.-H. Xiu and G. Ren, "Stability analysis and systematic design of Takagi-Sugeno fuzzy control systems," Fuzzy Sets and Systems, vol. 151, no. 1, pp. 119-138, 2005.

[8] C.-S. Ting, "Stability analysis and design of Takagi-Sugeno fuzzy systems," Information Sciences, vol. 176, no. 19, pp. 2817-2845, 2006.

[9] J. Yoneyama, "Robust stability and stabilization for uncertain Takagi-Sugeno fuzzy time-delay systems," Fuzzy Sets and Systems, vol. 158, no. 2, pp. 115-134, 2007.

[10] C.-C. Fuh, "Absolute stability analysis for a class of TakagiSugeno fuzzy control systems," International Journal of Systems
Science: Principles and Applications of Systems and Integration, vol. 39, no. 5, pp. 477-486, 2008.

[11] M. Bernal and T. Guerra, "Generalized nonquadratic stability of continuous-time Takagi-Sugeno models," IEEE Transactions on Fuzzy Systems, vol. 18, no. 4, pp. 815-822, 2010.

[12] X. Zhang, C. Wang, D. Li, X. Zhou, and D. Yang, "Robust stability of impulsive Takagi-Sugeno fuzzy systems with parametric uncertainties," Information Sciences, vol. 181, no. 23, pp. 52785290, 2011.

[13] O. M. Kwon, M. J. Park, S. M. Lee, and J. H. Park, "Augmented Lyapunov-Krasovskii functional approaches to robust stability criteria for uncertain Takagi-Sugeno fuzzy systems with timevarying delays," Fuzzy Sets and Systems, vol. 201, pp. 1-19, 2012.

[14] D. H. Lee, Y. H. Joo, and M. H. Tak, "Linear matrix inequality approach to local stability analysis of discrete-time TakagiSugeno fuzzy systems," IET Control Theory \& Applications, vol. 7, no. 9, pp. 1309-1318, 2013.

[15] Y. Manai and M. Benrejeb, "Stability analysis of time-delay Takagi-Sugeno fuzzy system: a new fuzzy Lyapunov-Razumikhin function," International Review of Automatic Control, vol. 5, no. 1, pp. 81-87, 2013.

[16] A. Benzaouia and R. Oubah, "Stability and stabilization by output feedback control of positive Takagi-Sugeno fuzzy discretetime systems with multiple delays," Nonlinear Analysis: Hybrid Systems, vol. 11, pp. 154-170, 2014.

[17] V. Bevelevich, Classical Network Synthesis, Van Nostrand, New York, NY, USA, 1968.

[18] G. Calcev, R. Gorez, and M. De Neyer, "Passivity approach to fuzzy control systems," Automatica, vol. 34, no. 3, pp. 339-344, 1998.

[19] L. Xie, M. Fu, and H. Li, "Passivity analysis and passification for uncertain signal processing systems," IEEE Transactions on Signal Process, vol. 46, no. 9, pp. 2394-2403, 1998.

[20] L. O. Chua, "Passivity and complexity," IEEE Transactions on Circuits and Systems. I: Fundamental Theory and Applications, vol. 46, no. 1, pp. 71-82, 1999.

[21] C. W. Wu, "Synchronization in arrays of coupled nonlinear systems: passivity, circle criterion, and observer design," IEEE Transactions on Circuits and Systems. I: Fundamental Theory and Applications, vol. 48, no. 10, pp. 1257-1261, 2001.

[22] S.-I. Niculescu and R. Lozano, "On the passivity of linear delay systems," IEEE Transactions on Automatic Control, vol. 46, no. 3, pp. 460-464, 2001.

[23] E. Fridman and U. Shaked, "On delay-dependent passivity," IEEE Transactions on Automatic Control, vol. 47, no. 4, pp. 664669, 2002.

[24] M. S. Mahmoud and A. Ismail, "Passivity and passification of time-delay systems," Journal of Mathematical Analysis and Applications, vol. 292, no. 1, pp. 247-258, 2004.

[25] C. Li and X. Liao, "Passivity analysis of neural networks with time delay," IEEE Transactions on Circuits and Systems II, vol. 52, no. 8, pp. 471-475, 2005.

[26] J. H. Park, "Further results on passivity analysis of delayed cellular neural networks," Chaos, Solitons and Fractals, vol. 34, no. 5, pp. 1546-1551, 2007.

[27] H. Gao, T. Chen, and T. Chai, "Passivity and passification for networked control systems," SIAM Journal on Control and Optimization, vol. 46, no. 4, pp. 1299-1322, 2007.

[28] E. M. Navarro-López and E. Fossas-Colet, "Feedback passivity of nonlinear discrete-time systems with direct input-output link," Automatica, vol. 40, no. 8, pp. 1423-1428, 2004. 
[29] G. Calcev, R. Gorez, and M. De Neyer, "Passivity approach to fuzzy control systems," Automatica, vol. 34, no. 3, pp. 339-344, 1998.

[30] C. Li, H. Zhang, and X. Liao, "Passivity and passification of fuzzy systems with time delays," Computers \& Mathematics with Applications, vol. 52, no. 6-7, pp. 1067-1078, 2006.

[31] B. Zhang, W. X. Zheng, and S. Xu, "Passivity analysis and passive control of fuzzy systems with time-varying delays," Fuzzy Sets and Systems, vol. 174, pp. 83-98, 2011.

[32] Q. Song, Z. Wang, and J. Liang, "Analysis on passivity and passification of T-S fuzzy systems with time-varying delays," Journal of Intelligent \& Fuzzy Systems: Applications in Engineering and Technology, vol. 24, no. 1, pp. 21-30, 2013.

[33] Q. Song, Z. Zhao, and J. Yang, "Passivity and passification for stochastic Takagi-Sugeno fuzzy systems with mixed timevarying delays," Neurocomputing, vol. 122, pp. 330-337, 2013.

[34] X. Liu, "Passivity and passification analysis of uncertain discrete-time fuzzy systems," Kybernetes, vol. 38, no. 3-4, pp. 396-405, 2009.

[35] G. Duan and Y. Li, "Robust passive control for discrete-time T-S fuzzy systems with delays," Journal of Systems Engineering and Electronics, vol. 20, no. 5, pp. 1045-1051, 2009.

[36] J. Liang, Z. Wang, and X. Liu, "On passivity and passification of stochastic fuzzy systems with delays: the discrete-time case," IEEE Transactions on Systems, Man, and Cybernetics B, vol. 40, no. 3, pp. 964-969, 2010.

[37] F. Ren and J. Cao, "Novel $\alpha$-stability criterion of linear systems with multiple time delays," Applied Mathematics and Computation, vol. 181, no. 1, pp. 282-290, 2006.

[38] J. Cao and J. Wang, "Delay-dependent robust stability of uncertain nonlinear systems with time delay," Applied Mathematics and Computation, vol. 154, no. 1, pp. 289-297, 2004.

[39] H. Xiang and J. Cao, "Periodic oscillation of fuzzy CohenGrossberg neural networks with distributed delay and variable coefficients," Journal of Applied Mathematics, vol. 2008, Article ID 453627, 18 pages, 2008.

[40] Y. Cao and Z. Lin, "Robust stability analysis and fuzzy-scheduling control for nonlinear systems subject to actuator saturation," IEEE Transactions on Fuzzy Systems, vol. 11, no. 1, pp. 57-67, 2003. 


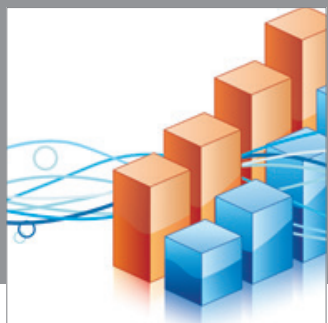

Advances in

Operations Research

mansans

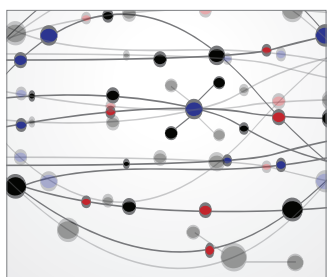

The Scientific World Journal
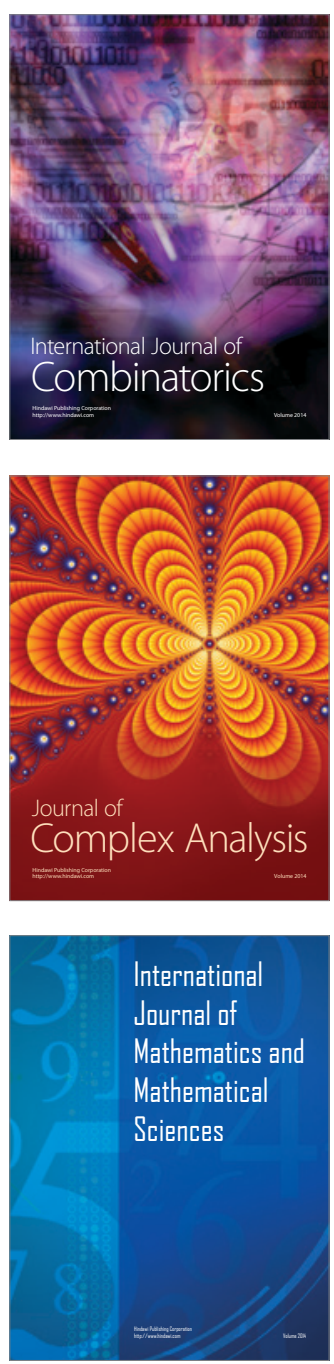
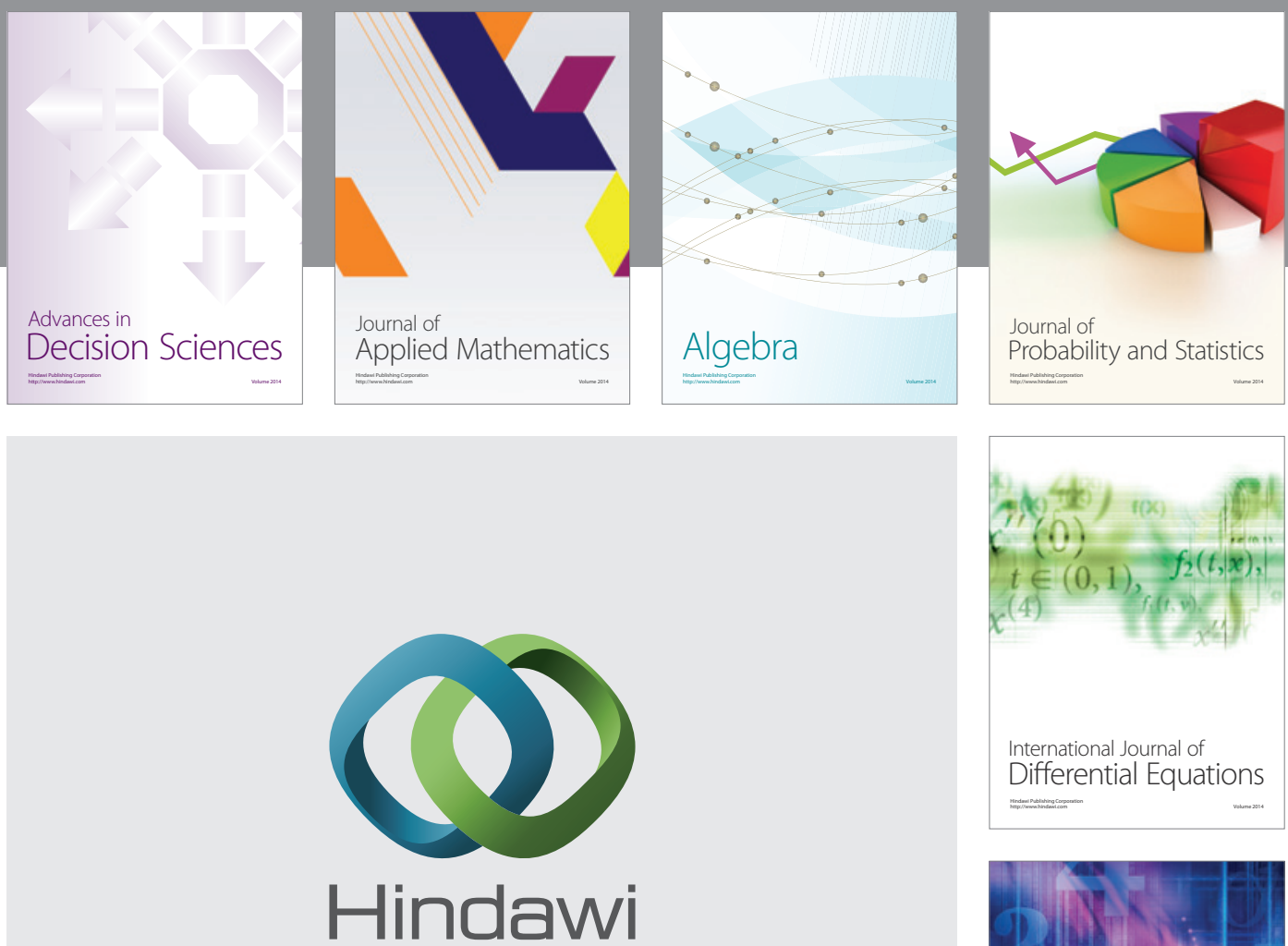

Submit your manuscripts at http://www.hindawi.com
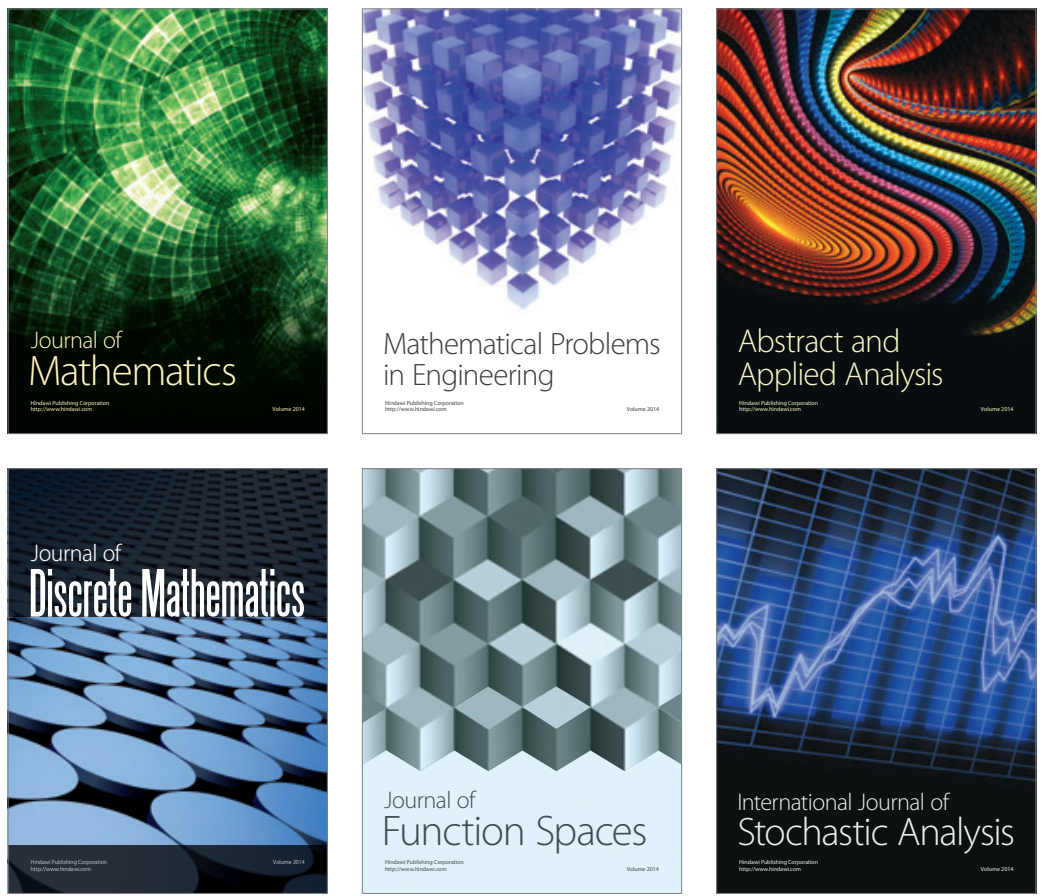

Journal of

Function Spaces

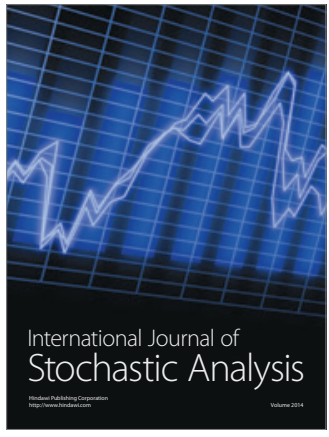

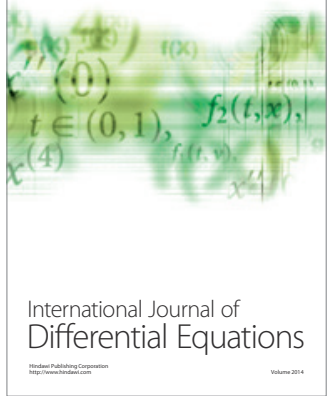
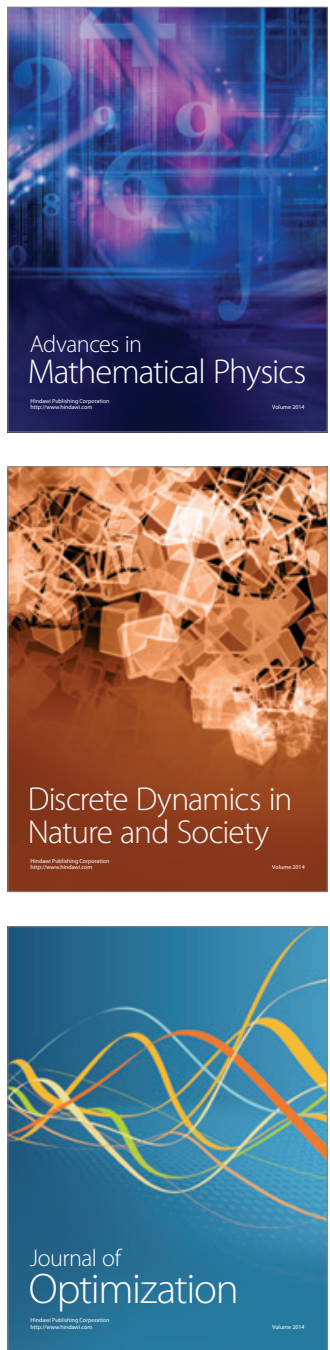